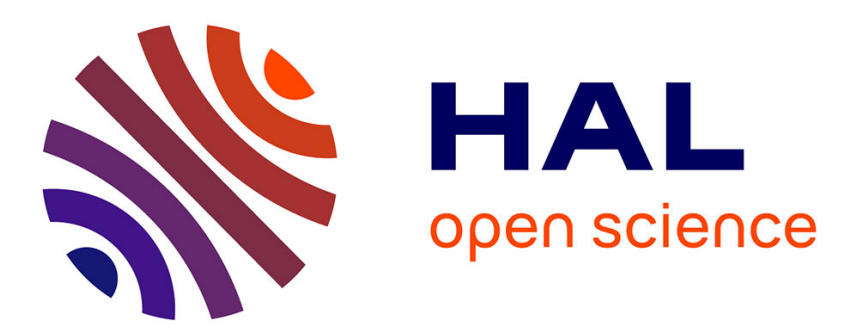

\title{
Order-Disorder Structural Transitions in Mazes Built by Evaporating Drops
}

Pascal Panizza, H. Algaba, M. Postic, Guillaume Raffy, Laurent Courbin, Franck Artzner

\section{- To cite this version:}

Pascal Panizza, H. Algaba, M. Postic, Guillaume Raffy, Laurent Courbin, et al.. Order-Disorder Structural Transitions in Mazes Built by Evaporating Drops. Physical Review Letters, 2018, 121 (7), pp.078002. 10.1103/PhysRevLett.121.078002 . hal-01879727

\section{HAL Id: hal-01879727}

https://hal-univ-rennes1.archives-ouvertes.fr/hal-01879727

Submitted on 13 Dec 2018

HAL is a multi-disciplinary open access archive for the deposit and dissemination of scientific research documents, whether they are published or not. The documents may come from teaching and research institutions in France or abroad, or from public or private research centers.
L'archive ouverte pluridisciplinaire HAL, est destinée au dépôt et à la diffusion de documents scientifiques de niveau recherche, publiés ou non, émanant des établissements d'enseignement et de recherche français ou étrangers, des laboratoires publics ou privés. 


\title{
Order-disorder structural transitions in mazes built by evaporating drops
}

\author{
P. Panizza, ${ }^{*}$ H. Algaba, M. Postic, G. Raffy, L. Courbin, ${ }^{\dagger}$ and F. Artzner \\ Univ Rennes, CNRS, IPR (Institut de Physique de Rennes) - UMR 6251, F-35000 Rennes, France
}

\begin{abstract}
We show that the evaporation of surfactant solutions confined in quasi two-dimensional (2D) porous media creates micron-sized labyrinthine patterns composing the walls of a centimeter-sized maze. These walls are made of solid deposits formed during drying via a sequence of individual Haines jumps occurring at the pore scale. We rationalize this process driven by simple iterative rules with a cellular automaton that acts as a maze generator. This model well describes the formation dynamics and final structure of an experimental maze as functions of the wettability heterogeneities of a porous medium and its geometry. Experiments and modeling work also unveil the crucial role of a geometric dimensionless quantity controlling the structural order of a maze.
\end{abstract}

PACS numbers: 47.56.+r 47.57.-s 47.50.Gj

Evaporation is central to many industrial and natural processes [1]. Understanding the evaporation of liquid dispersions in porous media is important for various disciplines, e.g., soil physics [2, 3] and civil engineering [4], and diverse processes such as the underground sequestration of $\mathrm{CO}_{2}$ [5]. The key issue in most cases is to comprehend the creation, locus and morphology of the solid deposits formed during drying. For instance, this topic is crucial in civil engineering as salt weathering can cause severe damage to the built environment [6] and in material science as evaporation can help the design of novel smart materials [7]. Yet, the development of models for drying in porous media [8] and the proper description of the formation of deposits in such media $[9,10]$ have remained elusive as these tasks are generally challenging.

Here, we study the evaporation of surfactant solutions in porous media consisting of micron-sized cylindrical posts arranged on square or rectangular lattices. The manufactured evaporation devices are transparent and quasi two-dimensional (2D). These two essential attributes allow us to precisely determine how and where solid deposits form during drying. As a fluid evaporates, labyrinthine patterns made of solid deposits form in the pores between adjacent posts. These 'walls' compose a centimeter-sized structure that resembles a maze when drying ends. Labyrinthine patterns can also emerge in fluid-granular 2D flows [11] and other fluid systems submitted to an external field [12]. Phyllotaxy-inspired drying patterns can even be seen in suitably designed $2 \mathrm{D}$ porous media [13]. In our study, we show that the created walls result from the motion of unstable liquid-air menisci between neighboring posts, i.e., Haines jumps. These events being sequential and stochastic at the lattice scale and driven by iterative rules at the pore scale, we rationalize our results with a cellular automaton generating mazes. This model, which accounts for pore-scale heterogeneities of wettability known to impact drying in porous media [14, 15], well describes the structure and formation dynamics of the observed mazes. For fixed wetting conditions, we show that the structural order of a maze is governed by only two geometric dimensionless quantities built with four key geometric parameters, the post radius, the lattice height and inter-post spacings.

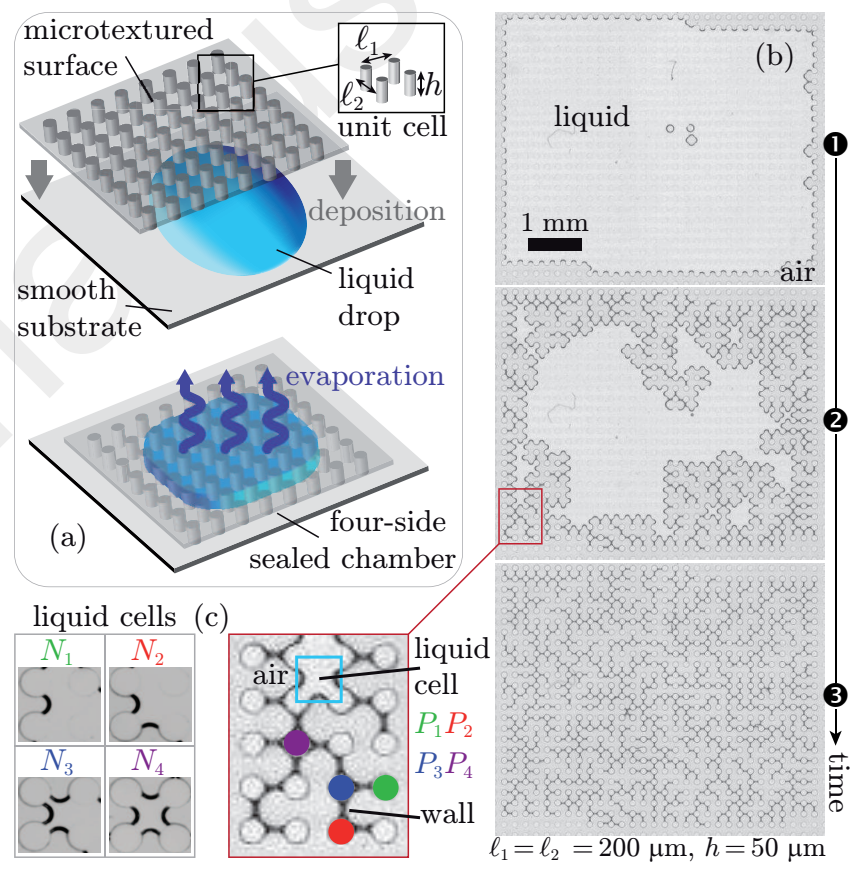

FIG. 1. (a) Schematics describing our experiment. (b) Topview images of an evaporating drop initially comprising 665 liquid cells. The corresponding times are as follows: (1) $t=$ $0 \mathrm{~s}$, (2) $t=8040 \mathrm{~s}$, and (3) $t=26600 \mathrm{~s}$. (c) Images of the liquid cells $N_{k}$ having $k$ menisci and close-up view of the red box shown in (b) defining the posts $P_{k}$ connected to $k$ walls.

An evaporation device consists of an assembly of posts sealed on top of a smooth solid (see Fig. 1), both surfaces being made of transparent poly-dimethylsiloxane (PDMS Sylgard 184, Dow Corning) using soft lithography [16]. The posts have a radius $R=50 \mu \mathrm{m}$ and height $h=20-100 \mu \mathrm{m}$, the inter-post spacings $\ell_{1}$ and $\ell_{2}$ being in 
the $150-250 \mu \mathrm{m}$ range [Fig. 1(a)]. A drop of a deionized water-surfactant (either SDS or Tween 20, concentration $5 \mathrm{cmc}$, Sigma-Aldrich) mixture is squeezed between textured and smooth surfaces and glue seals the sides of the device. The liquid then pervaporates $[17,18]$ through the ceiling of the device placed in a container with constant temperature and humidity [Fig. 1(a-b)]. For the whole study, experimental and numerical drops begin to evaporate at the origin of time $t=0$.

We use an inverted microscope (IX71, Olympus) and a camera (Eurocam) to record the evolution with time of the surface of an evaporating drop (Fig. 1(b), see [20] for further details on experimental methods). In an evaporation device, the liquid-air interface consists of circular menisci connecting adjacent posts (see Figs. 1-2). As the surface of a drop decreases during drying, all menisci slowly recede with a decreasing radius of curvature $R_{c}$ [Fig. 2(a)] until one of them becomes unstable and moves rapidly towards the liquid cell it contacts (see Fig. 2(b) and movie S1 in [20]); this is a Haines jump. As a result, new menisci or solid films or both are created at the three other edges of this cell (see Figs. 1(c)-2(b) defining the types of posts $P_{k}$ and liquid cells $N_{k}$ and illustrating the time evolution of these cells). A wall is built between two neighboring posts when liquid gets trapped between the moving unstable meniscus merging with another meniscus located on one edge of the invaded liquid cell [Fig. 2(b)]. The evaporation of this liquid bridge creates a solid wall and possibly the crystallization of the dispersed phase as illustrated by X-ray scattering and scanning electron microscopy when a nanorod-based fluid system is used [19]. Our experiments show that (i) the contact angle $\theta$ that the liquid makes with PDMS is $\theta_{m} \leq \theta \leq \theta_{M}$ with typically $\theta_{m} \simeq 25^{\circ}$ and $\theta_{M} \simeq 65^{\circ}$ (see Fig. S1 and related discussion in [20]), (ii) each meniscus presents an axis of symmetry perpendicular to the line connecting the centers of the two posts it contacts [see Fig. 2(a)] and (iii) $\theta$ is unchanged during the motion of an unstable meniscus. Also revealed by our observations, the selection of unstable menisci is stochastic and their motions are sequential, i.e., they move one at a time. Hence, evaporation consists of successive Haines jumps separated by slowly evolving configurations (see movie S1 in [20]) until the process ends. The interconnected walls then form a centimeter-sized structure resembling a maze [Fig. 1(b) and Fig. 2(d)]. Being seen for different solute species (surfactants and colloids), these structures are inherent to the drying process. To study their formation dynamics and topology, we characterize the temporal evolution of $P_{k}(t)$ and $N_{k}(t)$ by processing images recorded during drying with a custom-written C++ software.

We begin to rationalize our results by considering that $\theta$ is constant $\left(\theta=45^{\circ}\right)$ as this simplified analysis helps to understand the underlying physics of our experiment. $\mathrm{A} \ell_{i}$-meniscus and a $\ell_{i}$-wall $(i=1$ or 2$)$
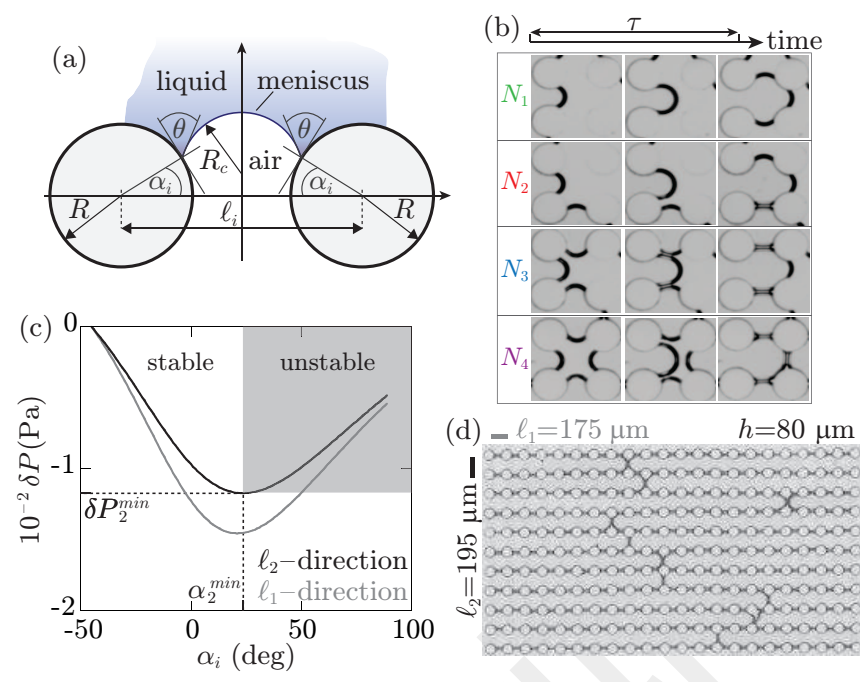

FIG. 2. (a) Top-view schematic of a meniscus connecting two adjacent posts defining geometric variables at play. (b) Photographs showing the four types of Haines jumps responsible for the time evolution of the liquid cells $N_{k}$ and subsequent creation of walls. The loss of a cell $N_{k}$ after a time $\tau$ produces $4-k$ new menisci and $k-1$ walls. (c) $\delta P$ versus $\alpha_{i}$ for $\ell_{1}=175 \mu \mathrm{m}$ (grey) and $\ell_{2}=195 \mu \mathrm{m}$ (black). $\theta=45^{\circ}$ and $h=80 \mu \mathrm{m}$. The shaded area indicates the unstable region in the $\ell_{2}$-direction. (d) Maze obtained with a rectangular lattice having geometrical parameters as in Fig. 2(b).

herein denote a meniscus and a wall connecting two adjacent posts with inter-post spacing $\ell_{i}$, respectively. Using geometry, one easily determines the radius of curvature $R_{c}=\frac{\ell_{i}-2 R \cos \alpha_{i}}{2 \cos \left(\theta-\alpha_{i}\right)}$ of a $\ell_{i}$-meniscus with $\alpha_{i}$ the angle defining its position on the posts it contacts [see Fig. 2(a)]. The pressure at this meniscus is $P\left(\alpha_{i}, \ell_{i}, h\right)=$ $P_{0}+\delta P$ with the ambient pressure $P_{0}$ and Laplace pressure $\delta P=-\gamma\left(R_{c}^{-1}+2 h^{-1} \cos \theta\right), \gamma$ being the air-liquid surface tension [21]. Figure 2(c) shows that $\delta P$ has a minimum $\delta P_{i}^{\text {min }}$ for $\alpha_{i}=\alpha_{i}^{\text {min }}$. Also, for any $h$, we find that $\delta P_{2}^{\text {min }} \geq \delta P_{1}^{\text {min }}$ when $\ell_{2} \geq \ell_{1}$. As shown below in the case $\ell_{2}>\ell_{1}$, these basic arguments are sufficient to explain the observed Haines jumps; note that similar arguments can be employed when $\ell_{2}<\ell_{1}$.

Between two successive Haines jumps, $\delta P$ is uniform so that $\alpha_{1}$ and $\alpha_{2}$ are small and different [see Fig. 2(c)]. As water evaporates, these two angles increase as $\delta P$ decreases until it reaches the value $\delta P_{2}^{\min }$ [Fig. 2(c)]. Then, if a fluctuation causes a $\ell_{i}$-meniscus to move farther than the others, the pressure at this meniscus either decreases $(i=1)$ or increases $(i=2)$ [Fig. 2(c)]. Hence, the pressure gradient between this local pressure and that of other liquid cells induces a flow that either stabilizes $(i=1)$ or destabilizes $(i=2)$ the moving front. All $\ell_{2}$-menisci are then potentially unstable. By contrast, all menisci are stable when $\delta P>\delta P_{2}^{\min }$ and $\alpha_{i}<\alpha_{i}^{\text {min }}$ since $\delta P$ decreases with $\alpha_{i}$ for both $i=1$ and 2 . Thus, all 
menisci should recede slowly at a speed set by the evaporation rate when $\delta P>\delta P_{2}^{\text {min }}$ until a Haines jump occurs for $\delta P=\delta P_{2}^{\text {min }}$. The motion of an unstable $\ell_{2}$-meniscus induces a rapid transfer of liquid in the other cells which makes all other menisci advance. So, once a Haines jump is completed, the pressure $\delta P>\delta P_{2}^{\text {min }}$ is uniform and all menisci are stable again. They recede slowly until another Haines jump occurs when $\delta P=\delta P_{2}^{\text {min }}$.
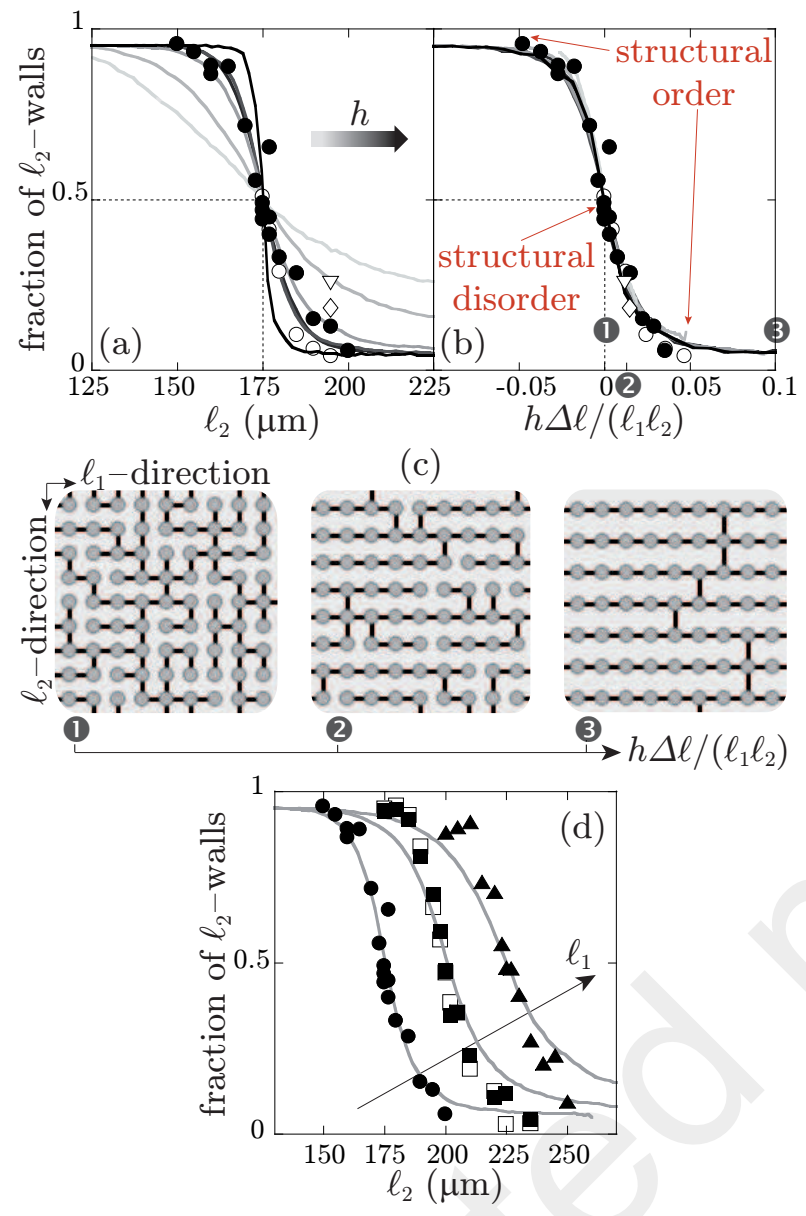

FIG. 3. (a) Experimental fraction of $\ell_{2}$-walls vs. $\ell_{2}$ for $\ell_{1}=175 \mu \mathrm{m}$ and four post heights $h: 20 \mu \mathrm{m}(\nabla), 25 \mu \mathrm{m}(\diamond)$, $50 \mu \mathrm{m}(\bullet)$ and $80 \mu \mathrm{m}(\circ)$. The lines correspond to our model with $\theta_{m}=25^{\circ}$ and $\theta_{M}=65^{\circ}$. As indicated by the arrow, the shades of gray of the lines stand for different $h$, varying from the lightest gray for the smallest post $(10 \mu \mathrm{m})$ to black for the tallest one $(200 \mu \mathrm{m})$ with intermediate values $20 \mu \mathrm{m}$, $50 \mu \mathrm{m}, 80 \mu \mathrm{m}$ and $100 \mu \mathrm{m}$. (b) Data shown in Fig. 3(a) vs. $\frac{h \Delta \ell}{\ell_{1} \ell_{2}}$. (c) Close-up views of a few tens of pores depicting the final numerical structures for different $\frac{h \Delta \ell}{\ell_{1} \ell_{2}}$; when a simulation starts, a drop comprises 400 liquid cells. (d) Fractions of $\ell_{2}$-walls vs. $\ell_{2}$ for $h=50 \mu \mathrm{m}$ and different $\ell_{1}$ : $175 \mu \mathrm{m}(\bullet)$, $200 \mu \mathrm{m}(\boldsymbol{\square}, \square)$ and $225 \mu \mathrm{m}(\boldsymbol{\Delta})$. The three lines are the outcomes of the cellular automaton for the water-SDS mixture with $\theta_{m}$ and $\theta_{M}$ as in Fig. 3(a). The open squares denote experiments conducted with the water-Tween 20 mixture.

Our analysis explains the observed evaporation charac- terized by a succession of individual Haines jumps. These stochastic events are periodic, their period $\tau$ being the time needed for a liquid cell to evaporate [see Fig. 2(b)]. As depicted in Fig. 2(b), a Haines jump yields the creation of new menisci or walls or both positioned along the edges of the invaded liquid cell according to simple rules. A wall is formed only when the unstable meniscus merges with another meniscus initially present on an edge of this liquid cell; a new meniscus is created otherwise. The final configuration of an invaded cell thus only depends its initial one observed just before the jump starts [Fig. 2(b)]. Within our approximation of constant angle $\theta$, only $\ell_{2}$ menisci are unstable during drying when $\ell_{2}>\ell_{1}$. The structure of a maze should then be highly ordered with only one $\ell_{2}$-wall formed per column of liquid cells in the $\ell_{1}$-direction. The final structure shown in Fig. 2(d) concur with this prediction that should neither depend on $h$ nor the lattice anisotropy $\Delta \ell=\ell_{2}-\ell_{1}$ when $\Delta \ell \neq 0$. For square lattices $(\Delta \ell=0)$, the whole picture is not altered. However all $\ell_{i}$-menisci are potentially unstable in this case so that the fraction of $\ell_{1}$-walls should be equal to that of $\ell_{2}$-walls. This is qualitatively confirmed by the final structure of the maze shown in Fig. 1(b).

To validate our predictions quantitatively, we perform experiments in which we vary $\Delta \ell$ and $h$ for a fixed $\ell_{1}$. As predicted, the same number of walls is found in the two directions of the lattice for any $h$ when $\Delta \ell=0$ and the fraction of $\ell_{2}$-walls is close to 0 when $\Delta \ell>20 \mu \mathrm{m}$ [see Fig. 3(a)]. Unexpectedly, however, a large $(\approx 0.1-0.4)$ fraction of $\ell_{2}$-walls is found experimentally for intermediate $\Delta \ell$ at any $h$ [Fig. 3(a)]; this fraction decreases with increasing $\Delta \ell$ (at fixed $h$ ) and $h$ (for a given $\Delta \ell$ ).

Although our simple analysis based on a unique contact angle helps to understand some results, it fails to explain the existence of unstable $\ell_{1}$-menisci yielding the finite fraction of $\ell_{2}$-walls seen for intermediate $\Delta \ell$. To that end, the contact angle is $\theta_{m} \leq \theta \leq \theta_{M}$ for the remainder of the study. For this problem driven by the iteration of simple rules, we develop a cellular automaton that acts as a maze generator to rationalize our experiments. This numerical model proceeds as follows. Each pore of a lattice is filled with either liquid or air and menisci connect the two types of cells. At each time step of the algorithm, we count the number $M_{i}$ of $\ell_{i}$-menisci, we index their positions for both $i=1$ or 2 with an integer $1 \leq j_{i} \leq M_{i}$ and we randomly select a contact angle $\theta_{m} \leq \theta_{j_{i}} \leq \theta_{M}$ for each of them [22]. At each $\ell_{i}$-meniscus, the excess pressure in the liquid is now $\delta P\left(\theta_{j_{i}}, \alpha_{i}, \ell_{i}, h\right)$. Similar to our previous analysis assuming a constant angle [see Fig. 2(b)], as menisci move slowly between two Haines jumps, $\delta P$ is uniform and decreases until it reaches the minimum value $\delta P_{i}^{\min }\left(\theta_{j_{i}}, \ell_{i}, h\right)=\min \left(\delta P\left(\theta_{j_{i}}, \alpha_{i}, \ell_{i}, h\right)\right)$ of the pressure profile at one meniscus. This minimum being the largest of all pressure profiles, the identified meniscus is unstable and the algorithm makes it invade its neighbor- 

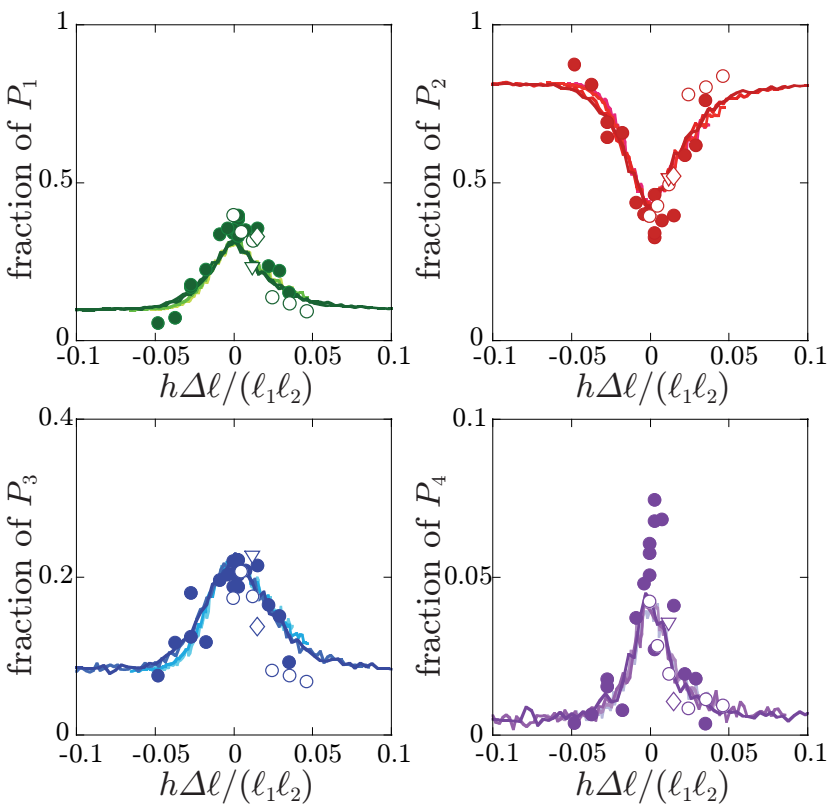

FIG. 4. Final fractions of $P_{k}$ vs. $\frac{h \Delta \ell}{\ell_{1} \ell_{2}}$ for $\ell_{1}=175 \mu \mathrm{m}$ $\left(\ell_{1} / R=3.5\right)$ and four post heights. The symbols are identical to those of Fig. 3(a). The lines are the outcomes of the cellular automaton with $\theta_{m}=25^{\circ}$ and $\theta_{M}=65^{\circ}$; the shades of color of the lines stand for the four heights varying from the lightest shade for the smallest post to the darkest shade for the tallest post.

ing cell according to the rules depicted in Fig. 2(b).

To compare model predictions with experiments, the initial shape of a squeezed drop and time step $\tau$ used in simulations match experimental ones. As shown in Fig. 3(a), the model accounting for wettability heterogeneities captures quantitatively well the experimental features that our simple analysis with a unique contact angle $\theta$ failed to describe. This model indeed explains the non-zero fractions of $\ell_{2}$-walls measured for $0<\Delta \ell<20 \mu \mathrm{m}$, fractions that decrease with increasing both $\ell_{2}$ (at fixed $h$ ) and $h$ (at fixed $\ell_{2}$ ).

Interestingly, experimental and numerical data collapse onto a master curve when plotted as a function of $\frac{h \Delta \ell}{\ell_{1} \ell_{2}}$ [Fig. 3(b)]. Besides the length scales involved in the numerator of this dimensionless quantity, it may be noted that this expression $\left(\propto \frac{1}{\ell_{1} \ell_{2}}\right)$ is similar to that of the wetted solid fraction of posts by a drop suspended on a rectangular array of posts [23]; in [20], we discuss the physical origin of this quantity in further details. The structural order of a maze appears to be an increasing function of this parameter as illustrated by the numerical mazes shown in Fig. 3(c). In addition, our model predicts well the variations of the fraction of $\ell_{2}$-walls when $\ell_{1}$ varies and final values of the fractions of $P_{k}$ (see Fig. 4) and our experiments seem to be independent of the nature of the surfactant [see Fig. 3(d)]. In our study with four geometric parameters $\left(h, \ell_{1}, \ell_{2}, R\right)$ at play, figure 3(b) reveals the governing dimensionless quantity $\frac{h \Delta \ell}{\ell_{1} \ell_{2}}$. $R$ and $\ell_{1}$ being constant in this figure, $\ell_{1} / R$ is another governing quantity (see Fig. S2 and a discussion on these quantities in [20]). Hence, data reported for different $\ell_{1} / R$ in Fig. 3(d) cannot collapse onto a master curve as a function of $\frac{h \Delta \ell}{\ell_{1} \ell_{2}}$ (see also Fig. S2(b) in [20]). As shown in [20], our model also captures the formation dynamics of a maze, i.e. the temporal variations of $P_{k}(t)$ and $N_{k}(t)$, for square (Fig. S3 and movie S2) and rectangular (Fig. S4 and movie S3) lattices; as illustrated in Fig. S4 in [20], our analysis does not capture the details of their because of the stochastic nature of the Haines jumps at the origin of the formation of a maze.

To conclude, studying the evaporation of surfactant solutions in transparent 2D porous media, we unravel the mechanisms governing the formation of solid deposits in the micron-sized pores, these deposits composing the walls of a centimeter-sized maze. Interestingly, two posts of a maze being connected by one path only, these mazes are called 'perfect' and they are equivalent to a tree in graph theory. We model our findings with a cellular automaton accounting for wettability heterogeneities and acting as a maze generator that captures the structure of a created maze and its formation dynamics. Also, our findings unveil a geometrical dimensionless quantity that allows for a fine tuning of the structural order of a maze. These results could help the design of multiscale materials using evaporation as a route of controlled selfassembly. In particular, the design rules described here could find applications in flexible electronics as they offer possibilities for tailoring microwire networks.

Acknowledgments: This work was partially funded by the Direction Générale de l'Armement.

* pascal.panizza@univ-rennes1.fr

† laurent.courbin@univ-rennes1.fr

[1] W. Brutsaert, Evaporation into the Atmosphere: Theory, History and Applications (Springer, Dordrecht, 1982).

[2] A. S. Goudie, and H. A. Viles, Salt Weathering Hazards (Wiley, London, 1997).

[3] U. Nachshon, N. Weisbrod, M. I. Dragila, and A. Grader, Water Resour. Res. 47, W03513 (2011).

[4] F. G. Bell, Environ. Geol. 21, 187 (1993).

[5] Y. Peysson, M. Fleury, and V. Blasquez-Pascual, Transp. Porous Media 90, 1001 (2011).

[6] N. Shahidzadeh-Bonn, J. Desarnaud, F. Bertrand, X. Chateau, and D. Bonn, Phys. Rev. E 81, 066110 (2010).

[7] I. U. Vakarelski, D. Y. C. Chan, T. Nonoguchi, H. Shinto, and K. Higashitani, Phys. Rev. Lett. 102, 058303 (2009).

[8] For a review on models of drying in porous media, see for instance M. Prat, Chem. Eng. J. 86, 153 (2002).

[9] R. Du and H. A. Stone, Phys. Rev. E 53, 1994 (1996).

[10] S. Veran-Tissoires, M. Marcoux, and M. Prat, Phys. Rev. Lett. 108, 054502 (2012).

[11] B. Sandnes, H. A. Knudsen, K. J. Måløy, and E. G. Flekkøy, Phys. Rev. Lett. 99, 038001 (2007). 
[12] R. E. Rosensweig, M. Zhan, and R. Shumovich, J. Magn. Magn. Mater. 39, 127 (1983).

[13] C. Chen, P. Duru, P. Joseph, S. Geoffroy, and M. Prat, Sci. Rep. 7, 15110 (2017).

[14] H. Chraïbi, M. Prat, and O. Chapuis, Phys. Rev. E 79, 026313 (2009).

[15] O. Borgman, P. Fantinel, W. Lühder, L. Goehring, and R. Holtzman, Water Resour. Res. 53, 5645 (2017).

[16] D. C. Duffy, J. Cooper McDonald, O. J. A. Schueller, and G. M. Whitesides, Anal. Chem. 70, 4974 (1998).

[17] J. B. Salmon, and J. Leng, J. Appl. Phys. 107, 84905 (2010).

[18] N. Ziane, and J. B. Salmon, Langmuir 31, 7943 (2015).

[19] C. Hamon, M. Postic, E. Mazari, T. Bizien, C. Dupuis, P. Even-Hernandez, A. Jimenez, L. Courbin, C. Gosse, F. Artzner, and V. Marchi-Artzner, ACS Nano 6, 4137 (2012).
[20] See Supplemental Material at [] for details on experimental methods, typical contact angle distributions, a brief discussion on governing parameters, two figures comparing experimental and numerical formation dynamics for rectangular and square lattices and three movies. Movie S1 illustrates experimental Haines jumps and the resulting formation of walls. Movie S2 and movie S3 show that the experimental structures of a maze compare well to numerical predictions for both kinds of lattices.

[21] The surface tensions determined by pendant drop tensiometry are $35 \mathrm{mNm}^{-1}$ and $36 \mathrm{mNm}^{-1}$ for the SDSbased system and Tween 20-based one, respectively.

[22] We consider a typical length scale of wettability heterogeneities given by that of a meniscus and a rectangular distribution of contact angles $\theta$ for the sake of simplicity.

[23] A. Gauthier, M. Rivetti, J. Teisseire, E. Barthel, Phys. Rev. Lett. 110, 046101 (2013). 